\title{
UM OLHAR EM BUSCA DA EQUIDADE: PROMOÇÃO BUCAL AOS ADOLESCENTES DO CASE-SM ${ }^{1}$
}

\author{
THE LOOKING FOR EQUITY: \\ ORAL HEALTH PROMOTION IN ADOLESCENTS FROM CASE-SM
}

\author{
Alan Carvalho Daros ${ }^{2}$, Gabriela do Amaral Silveira ${ }^{3}$, Larissa Machado Saldanha ${ }^{4}$, \\ Luiza Noal Tonetto ${ }^{5}$, Abdel Rahim Mohamad Abdel Salam Suleiman ${ }^{6}$, \\ Aline Kruger Batista ${ }^{7}$ e Lenise Menezes Seerig ${ }^{8}$
}

\section{RESUMO}

O CASE- SM (centro de atendimento socioeducativo de Santa Maria - RS), atende, em regime de internação, adolescentes do sexo masculino, com idade entre de 12 a 18 anos. Possui capacidade para 40 internos e, no momento da atividade, contava com 75 meninos internados. É regulamentado pelo SINASE (Sistema Nacional de Atendimento Socioeducativo). A FASE é orientada pelos princípios da universalidade, acessibilidade, do vínculo, da continuidade do cuidado, da integralidade da atenção, da responsabilização, da humanização, da equidade e da participação social. Esta ação objetivou a transmissão de informações de sobre higiene bucal, conhecimentos de patologias, contágio, modo de prevenção, além de fatores comuns de risco, como álcool, drogas, IST (infecções sexualmente transmissíveis), hábitos (piercing), uso de aparelhos ortodônticos. O presente trabalho é um relato de vivência de uma ação de saúde bucal intersetorial realizada na escola estadual Humberto de Campos, a qual funciona nas dependências do CASE-SM e do curso de Odontologia da UFN, por meio da disciplina de ações em odontologia I. A ação aconteceu em três etapas, contemplando todos os adolescentes matriculados na escola, foi feita a apresentação em multimídia. Com essa experiência, foi possível perceber o que é falado de equidade em teoria, mas que na prática esquecemos que todos somos seres humanos, seres errantes, que se arrependem e merecem uma segunda chance. Desse modo, essa experiência mostrou-nos diferentes maneiras de como trabalhar estes preconceitos e desmistificá-los, possibilitando que a formação em Odontologia seja reflexiva e crítica, como preconizado pelas diretrizes de educação.

Palavras-chave: Promoção de Saúde Bucal; Medida Socioeducativa; Integralidade.

\section{ABSTRACT}

CASE-SM (socio-educational service center in Santa Maria - RS), attends male adolescents, from 12 to 18 years old, on an inpatient basis. It has a capacity for 40 inmates and at the moment had 75 inpatients. It is regulated by SINASE (National System of Social and Educational Assistance). The FASE is guided by the principles of universality, accessibility, bonding, continuity of care, comprehensive care, accountability, humanization, equity and social participation. This action aimed at transmitting information on oral hygiene, knowledge of pathologies, contagion, mode of prevention, in addition to common risk factors, such as alcohol,

1 Relato de Experiência.

2 Discente do curso de odontologia UFN. E-mail: alan.daros1@gmail.com

3 Discente do curso de odontologia UFN. E-mail: silveiragabriela9800@gmail.com

4 Discente do curso de odontologia UFN. E-mail: larissa.machado307@gmail.com

5 Discente do curso de odontologia UFN. E-mail: luizanoaltonetto@gmail.com

6 Discente do curso de odontologia UFN. E-mail: AbdelSuleiman55@gmail.com

7 Docente do curso de odontologia UFN. E-mail: aline.kruger@prof.ufn.edu.br

8 Docente do curso de odontologia UFN. E-mail: lenise.seerig@prof.ufn.edu.br 
drugs, STIs (sexually transmitted infections), habits (piercing), use of orthodontic appliances. The present work is an experience report of an intersectoral oral health action carried out at the state school Humberto de Campos, which works on the premises of CASE-SM and the Dentistry course at UFN) through the discipline of actions in dentistry I. The action took place in three stages, covering all adolescents enrolled at the school, the presentation was made in multimedia. With this experience, it was possible to understand what is said about equity in theory, but in practice we forget that we are all human beings, errant beings, who repent and deserve a second chance. In this way, this experience showed us different ways of working with these prejudices and demystifying them, making it possible for Dentistry training to be reflective and critical, as recommended by the education guidelines.

Key words: Oral Health Promotion; Socio-Educational measure; Integrality.

\section{INTRODUÇÃO}

O CASE- SM (centro de atendimento socioeducativo de Santa Maria - RS), é uma unidade da FASE-RS (Fundação de Atendimento Socioeducativo do Estado do Rio Grande do Sul), o qual recebe adolescentes do sexo masculino, com idade entre de 12 a 18 anos que cumprem medida socioeducativa de internação por ato infracional. O local possui capacidade para 40 internos e, à época da ação (ano de 2019), contava com 75 adolescentes cumprindo medida. A Fundação é regulamentada pelo SINASE (Sistema de Nacional de Atendimento Socioeducativo) que executa as medidas socioeducativas impetradas pelo poder Judiciário e destinadas a adolescentes que praticaram ato infracional (RIO GRANDE DO SUL, 2002). A medida de internação é considerada último recurso conforme o ECA (Estatuto da Criança e do Adolescente), e somente deve ser aplicada em situações de extrema gravidade, onde não há outra medida mais adequada, sendo sujeita aos princípios de brevidade e de excepcionalidade (BRASIL, 1990).

Segundo a Política Nacional de Atenção Integral à Saúde de Adolescentes em Conflito com a Lei, em Regime de Internação e Internação Provisória (PNAISARI) (BRASIL, 2014) os adolescentes em cumprimento de medida socioeducativa em meio aberto e fechado, possuem garantia de atenção à saúde no Sistema Único de Saúde (SUS), no que diz respeito à promoção, prevenção, assistência e recuperação da saúde, nas três esferas de gestão, possuindo tantos direitos de acesso à Saúde quanto o restante da população. Sendo assim, o CASE-SM conta com uma equipe multiprofissional composta por psicólogas, assistentes sociais, enfermeira, dentista, médico clínico geral, psiquiatra, educador físico, advogado e pedagogo, que fazem um acompanhamento diário e integral dos jovens. Essas equipes possuem o dever de elaborar e incentivar a articulação dos Projetos Terapêuticos Singulares aos Planos Individuais de Atendimento (PIA), previstos no Sistema Nacional de Atendimento Socioeducativo (Sinase), de modo a atender às complexas necessidades desta população, realizando uma escuta qualificada e assistência resolutiva aos problemas que podem ser apresentados (ZAPPE et al., 2013).

Estes acompanhamentos e elaboração de projetos são uma ferramenta na busca de garantia de equidade, visto que estes adolescentes encontram-se em um estado de maior vulnerabilidade. 
Os principais aspectos promotores de vulnerabilidade desses adolescentes são a própria condição de desenvolvimento psíquico em que se encontram (adolescência), o contexto social em que estão inseridos e a institucionalização (BRASIL, 2000). Dessa forma, torna-se imprescindível um olhar mais atento do indivíduo como um todo. Dentro desse contexto, a FASE é orientada pelos princípios da universalidade, acessibilidade, do vínculo, da continuidade do cuidado, da integralidade da atenção, da responsabilização, da humanização, da equidade e da participação social.

A equidade é um dos princípios ético-doutrinários que fornece a base ideológica do SUS e se expressa nas ações de cuidado no cotidiano dos profissionais nas unidades de saúde (CUNHA, 1998). Por meio dela, tentamos diminuir as diferenças sociais existentes, proporcionando atendimento desigual para necessidades desiguais, resolvendo problemas específicos, com atendimentos personalizados para essas determinadas causas, caracterizando-se como o princípio da justiça social (6). Buscando sempre a equidade podemos manejar todos os outros princípios melhorando assim, o acesso e atendimento para os indivíduos, considerando as peculiaridades de cada caso.

O presente trabalho é um relato de vivência de uma ação de saúde bucal intersetorial realizada na escola estadual Humberto de Campos, a qual funciona nas dependências do CASE-SM e do curso de Odontologia da Universidade Franciscana (UFN), por meio da disciplina de Ações Integradas em Odontologia I.

\section{METODOLOGIA}

Nesta disciplina, procura-se a realização de ações de saúde bucal em grupos de adolescentes, especialmente os em condições mais adversas e com menos acesso às ações de saúde. Nesta situação estão os adolescentes em regime de internação, onde muitas vezes, os profissionais de saúde (incluindo aqui os acadêmicos) são os únicos a poder levar algum momento de cuidado.

Em busca de uma maior aproximação dos estudantes com a realidade social, as disciplinas extensionistas possuem um plano de ensino que reúne os conhecimentos adquiridos em sala de aula com a prática fora dela. Desse modo, a ideia da intervenção foi pensada e formulada de uma forma que atingisse o maior número possível de internos e estudantes matriculados.

A metodologia utilizada nesta disciplina extensionista foi a Espiral Construtivista, proposta por Lima (2017), uma metodologia ativa de ensino-aprendizado que consiste em identificar os problemas, elaborar a questão problema, traçar objetivos, estabelecer hipóteses de explicação ou solução e aplicar à realidade. As atividades de extensão relatadas neste estudo ocorreram de abril a julho do ano 2019.

A ação aconteceu, com os estudantes do terceiro semestre do curso de odontologia da Universidade Franciscana sendo organizados em dois grupos diferentes, para que contemplassem todos os adolescentes matriculados na escola (turnos manhã e tarde). A ação se desenvolveu em etapas, onde na primeira etapa foi realizada uma apresentação em multimídia em aula expositiva-dialogada 
relacionada a saúde bucal, que enfatizava as principais doenças, entre elas a gengivite, periodontite e cárie. Nessa etapa, além de informações sobre essas doenças, os alunos conseguiram interagir com os adolescentes, demonstrando melhores formas de identificar essas possíveis enfermidades.

Na segunda etapa, foi realizada uma apresentação que tinha como enfoque o uso de piercings e instrução sobre maneiras corretas de higienização dos mesmos (Figura 1). Foram demonstradas imagens e vídeos para um melhor entendimento dos internos. Na terceira etapa a apresentação continha informações sobre o tabagismo, onde foi explicado desde doenças no trato-respiratório até o mascaramento de sinais de outros malefícios bucais, sendo explicado a eles o que o uso do tabaco pode acabar acarretando, desde a redução da resposta cicatricial até o dano das funções celulares defensivas contra os patógenos orais, podendo levar a maior facilidade de desenvolver problemas periodontais, e também a halitose.

Figura 1 - Acadêmicos de Odontologia e adolescentes internos do CASE-SM

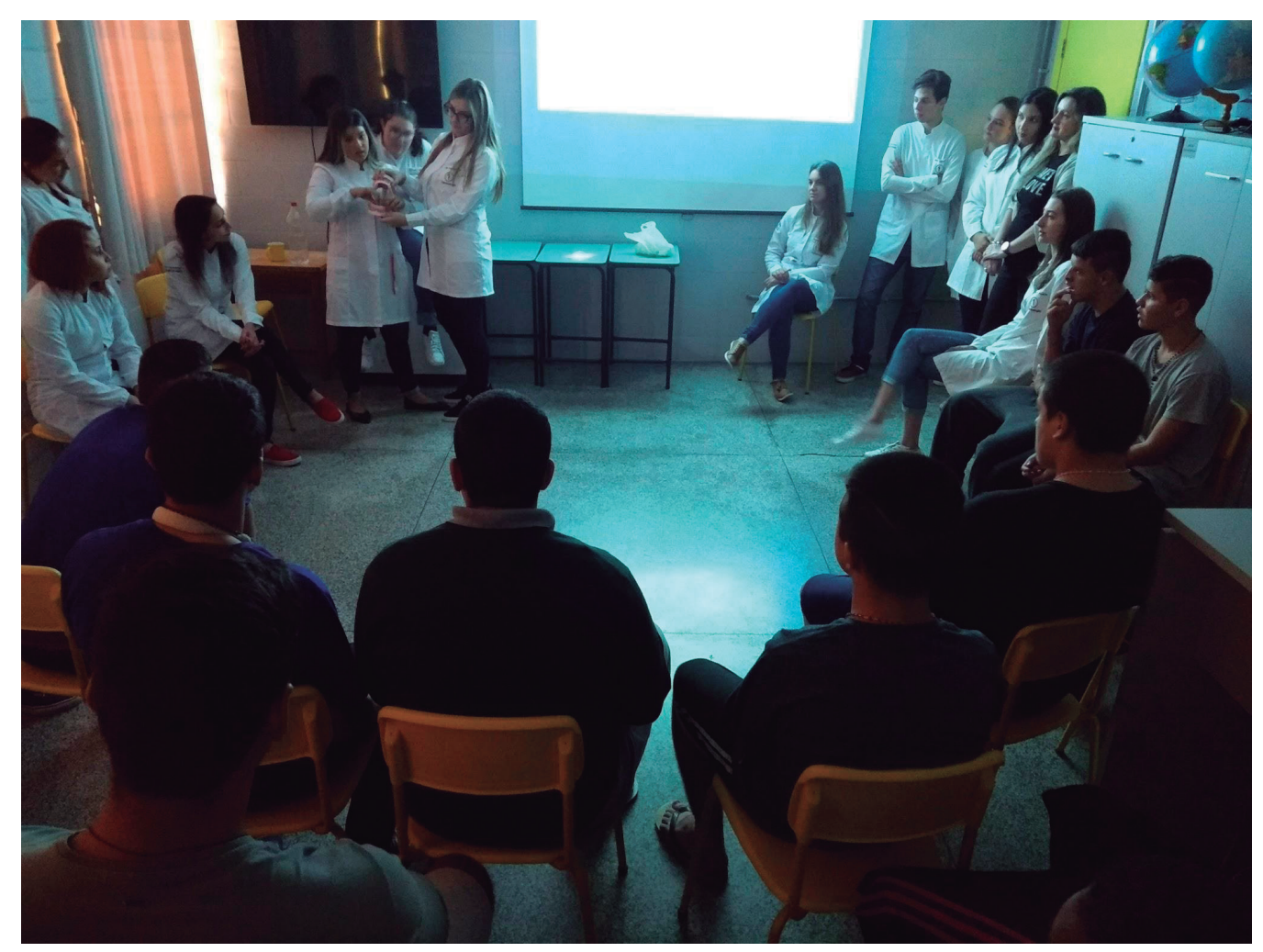

Fonte: acervo dos autores.

Na quarta e última etapa, foram utilizados macromodelos para a demonstração de uma correta escovação e uso de fio dental. Para concluir essas interações, jogos foram utilizados para fixação das informações passadas durante a aula e também com o intuito de formação de vínculo entre os acadêmicos e os adolescentes do Case-SM. Essa ação teve como objetivo levar os princípios de promoção e prevenção de saúde bucal e geral a este público em situação de vulnerabilidade e precariedade de acesso à saúde e informação. 


\section{RESULTADOS E DISCUSSÃO}

O CASE-SM tem capacidade para atender 40 adolescentes, no entanto, estava acomodando 75 pessoas, excedendo a sua capacidade máxima. As atividades na FASE foram realizadas em 4 etapas, com a divisão dos estudantes do curso de odontologia em dois grupos formados por 10 graduandos. Cada etapa abrangeu 25 alunos, que eram divididos pelos anos que estavam na escola, além disso, os alunos do turno da manhã eram do setor da CASE que podiam sair nos finais de semana - Internação com possibilidade de atividade externa (ICPAE), e as tardes eram de outro setor, que não saem nos finais de semana - Internação sem Possibilidade de Atividade Externa (ISPAE).

Buscando refletir sobre as diversas situações adversas da sociedade, a extensão universitária configura-se como uma das formas de mudança e apreensão da realidade social mais importantes, pois considera, o contexto social, político e econômico da população a ser assistida (SANTOS, 2012). Considerando a inserção da Extensão nos currículos dos cursos de graduação, a Universidade Franciscana (UFN) de Santa Maria - RS possui nove cursos de graduação na área da saúde, dentre estes o curso de Odontologia, o qual conta com seis disciplinas extensionistas. A disciplina de Ações Integradas em Odontologia I integra a base curricular do curso de Graduação em Odontologia, e é oferecida no terceiro semestre, tendo como base metodológica a espiral construtivista (LIMA, 2017).

Todas as atividades propostas tinham embasamento nas aulas teóricas sobre promoção de saúde. Os estudantes procuraram passar informações e agregar conhecimentos para os alunos, que através de mini aulas, questionários e brincadeiras, mostraram a importância do cuidado com eles mesmos. Além das questões relativas à saúde bucal procurou-se abordar outros comportamentos vulneráveis que poderiam trazer risco à saúde, como uso do álcool, drogas, fumo e iniciação sexual, corroborando com Gonçalves et al, 2015 que sugere que estratégias educativas e socioculturais em saúde devem ser praticadas desde o início da adolescência.

Ao conhecermos a parte física do CASE-SM notamos, que apesar da existência de propostas para tornar o acesso, mesmo que limitado aos adolescentes internos à saúde, escola e outras atividades extracurriculares, ainda existe um grande preconceito enraizado com os mesmos por parte da população externa. No caso dos acadêmicos de Odontologia, antes mesmo de adentrar no local, foi-nos passadas algumas instruções, como o modo de se vestir, acessórios que não eram permitidos - como brincos, colares, relógios e celulares, postura e o modo que devíamos ter e o cuidado com as coisas que devíamos falar. Tudo isso, juntamente com toda a carga de preconceito advinda do meio externo acabou deixando-nos com certa apreensão e medo.

Havia um certo anseio da nossa parte de não ter uma recepção favorável, porém ao passar do tempo houve uma grande surpresa, pois os meninos foram extremamente receptivos e aceitaram as propostas de trabalho apresentadas. Mostraram-se interessados e participativos durante todas as atividades propostas e ficaram ainda mais animados quando trouxemos brincadeiras 
com perguntas e ofertamos brindes, que mesmo sendo simples, foram vistos como algo muito valioso para eles.

Com essa experiência, foi possível perceber o quanto falar de equidade é necessário, mas além dessa teoria é preciso vivenciar realidades distintas das nossas, pois na prática devemos lembrar que somos seres humanos, passíveis de erros e de arrependimentos. Nem todo mundo tem as mesmas oportunidades ou advém dos mesmos contextos sociais. Ao tocar em outro ser humano, independente do local que se encontrem, devemos ter um olhar com maior equidade e compaixão, pois todos merecemos uma segunda chance.

\section{CONCLUSÃo}

Essa experiência mostrou-nos modos de como trabalhar estes preconceitos e desmistificá-los, possibilitando que a formação em Odontologia seja reflexiva e crítica, como preconizado pelas diretrizes curriculares e as propostas de extensão. Portanto, por mais que sejam meninos em regime socioeducativo de internação socioeducativo, independente do motivo, são pessoas possuem história, direitos e deveres, e cabe a nós, profissionais de saúde, ajudar na reintegração desses adolescentes à sociedade.

\section{REFERÊNCIAS}

BRASIL. Estatuto da Criança e do Adolescente - ECA. lei 8069, 13 de julho de 1990.

BRASIL, Ministério da Saúde. Política Nacional de Atenção Integral à Saúde de Adolescentes em Conflito com a Lei, em Regime de Internação e Internação Provisória (PNAISARI). Portaria $\mathrm{n}^{\circ} 1082$, de 23 de maio de 2014 .

BRASIL, Ministério da Saúde. Sistema Único de Saúde - SUS: princípios e conquistas, 2000

CUNHA, J. P. P.; Cunha, R. R. E. Sistema Único de Saúde - SUS: princípios. Cadernos de Saúde, Planejamento e Gestão em Saúde. Belo Horizonte: s.n., 1998.

GONÇALVES, Helen et al. Início da vida sexual entre adolescentes (10 a 14 anos) e comportamentos em saúde. Revista Brasileira de Epidemiologia, São Paulo, v. 18, n. 1, 2015.

LIMA, VV. Espiral construtivista: uma metodologia ativa de ensino-aprendizagem. Interface, Botucatu, v. 21, n. 61, p. 421-434, 2017. 
RIO GRANDE DO SUL. Assembleia Legislativa, dec 41.664, 2002.

SANTOS, M. P. dos. Extensão Universitária: Espaço de aprendizagem profissional e suas relações com o ensino e a pesquisa na educação superior. Revista Conexão, UEPG, v. 8, n. 2, p. 154-163, jul/dez. 2012.

ZAPPE, J. G.; SANTOS, C. R. dos; FERRÃO, I. da S.; DIAS, A. C. G. Vulnerabilidade e autonomia na pesquisa com adolescentes privados de liberdade. Psicologia: ciência e profissão, v. 33, n. 1, p. 234-247, 2013. 